\title{
Complexity Theory: Artificial Intelligence System Help Safety Improvement in the Next Pandemic
}

\author{
Shahad Faisal Halabi \\ University of Nottingham, Nottingham, The United Kingdom \\ Correspondence to: Shahad Faisal Halabi; Shahad.halabi@gmail.com
}

Received 08 August 2020;

Accepted 28 August 2020;

Published 01 September 2020

\begin{abstract}
As the coronavirus pandemic spread from Asia to the western world, drug discovery came to a near standstill. Most laboratories shut down and instruments and reagents were left untouched, except for the most essential work. The pandemic forced large and small companies, regulatory and government agencies, and academia to tap into technology, particularly artificial intelligence (AI) and machine learning (ML), for providing more than just speed and efficiency. This essay aims to dig deeply in complexity theory to help improve safety and reduce the impact of the next pandemic. It is based on implementing Artificial Intelligence (AI) to provide the safer complex theory with an example of the current situation of COVID-19. While there are no shortcuts around scientific rigor and experimentation, AI can certainly accelerate the discovery of new drugs particularly when combined with high-performance computing (HPC) and quantum computing. Evaluating new AI technologies, particularly in areas of drug discovery where there are few demonstrations of success, can be a real challenge. It is considered that safety improvement of alert systems and the risk factors, in order to organize the safety of health facilities and control the hospital environment before the potential pandemic develops. Here, we will try to apply complexity theory in our dealing with future pandemics based on the situation analysis of previous experiences.
\end{abstract}

\section{Introduction}

The purpose of this essay is to explore complexity theory to help improve safety and reduce the impact of the next pandemic. It is based on implementing Artificial Intelligence (AI) to provide the safer complex theory with an example of the current situation of COVID-19. It considers the safety improvement of alert systems and the risk factors, in order to organise the safety of health facilities and control the hospital environment before the potential pandemic develops.

\section{Background}

The general global view of complexity theory is that it is a middle range theory contributing to organizing, designing information systems, developing microsystems, and preparing for bioterrorism (Greenhalgh et al., 2010). Middle range theory classifies to nine middle theory appreciated as a significant structure of nursing knowledge for developing concept with multidisciplinary perspectives (Liehr and Smith, 2017). So, complexity theory is providing an explanation of structures and patterns in certain systems by restructure knowing outcomes at the global level (Paley and Eva, 2011). Also, complexity theory has the power to incorporated in many direction and variety of research design to scout multiple phenomena (Thompson et al., 2016). According to Sturmberg et al. and Ladyman et al. (2013), complexity theory is defined as a phenomenon of the dynamic and non-linear nature of the real-world system, called a Complex Adaptive System (CAS). It is a revolution in understanding system behaviour to control and predict, thus far, the world economy and the human brain.

A CAS is the connection between information, probability, and complexity (Hmelo-Silver and Pfeffer, 2004). According to Begun et al., (2003) a CAS has the ability to adjust a norm in a large human network to create a self-ordering behavioural structure. It helps the organization to survive different types of environmental conditions (Begun et al., 2003). While the CAS is the power of AI, applying these homogenous contexts to improve safety by predicting the hazard of the next influenza pandemic (Liu, 2018).

AI is a complexity theory approach with a wide range of uses. For example, in this paper it is used as a problem solving to measure and predict when, how and what the situation is within the next pandemic with basic information (Floridi, 2020). While intelligence is a style of thinking and behaving of a human or machine in a physical environment, AI mimics human behaviour and cognition (Jiang et al., 2017). Taking into consideration, preparation of the next influenza pandemic as example.

It is important to know the basic history of influenza pandemics, starting with the 'Mother of all Pandemics' was the first influenza pandemic H1N1 (Spanish flu), which started in 1918 and killed around 100 million people worldwide (Taubenberger and Morens, 2006). Passing to the current pandemic (COVID-19), which is reported in December 2019 with number of cases and deaths is still rising. 
Since 1952, the WHO's Global Influenza Surveillance and Response System inside of the Collaborating Centres and Essential Regulatory Laboratories has been overseeing influenza elements and the vaccine response (WHO, 2017). Usually, the worldwide prepared and designed an organisation to help respond to fires, bombs, earthquakes, and hurricanes, but what about a pandemic? As these natural disasters will happen and we predicted it, also pandemic will happen again and we could predict it. Planning predictable organisation for the pandemic could be the key to reducing the number of cases and deaths in hospitals (Shearer et al., 2020). Ensuring how and why things go right is related to Safety-II, which is a non-linear flexible system known to be complex (Hollnagel et al., 2015). Gradually, before the virus become a pandemic there was a lack of epidemic control or it was neglected in some stages.

The influenza pandemic is non-linear system and controlling this pandemic needs a complex theory. Not just theory, it needs an intelligence to predict the missing information to control and reduce the pandemic virus (Yassine and Shah, 2020). To build a norm of big information about virus and symptoms, which help researchers explore the pandemic to improve healthcare organization and provide safety development.

\section{The appreciation for a complex system in the influenza pandemic}

According to Langley et al. (2009), profound knowledge is important for improving activities like a complex system. Deming's system of profound knowledge, as shown in Figure 1, is classified into four parts. The relevant part of the complex system is 'appreciation of a system' (Langley et al., 2009). If we appreciate the knowledge of the complex system and use the CAS as an application of this knowledge, by predicting that $\mathrm{AI}$ is an integral part of complexity, we could successfully assess past influenza pandemics and prepare well for the next one by identifying the level of risk and how we can protect healthcare services.

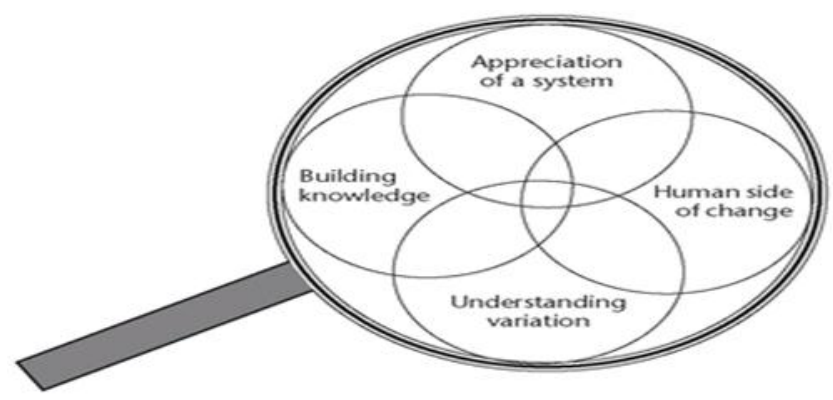

Figure 1: Deming's System (Langley et al., 2009)

'In complex projects, multiple measures are almost always needed to understand the impact of changes on the components of the system and on the system as a whole' (Langley et al., 2009:77).

The healthcare providers prepare AI as a complex system to help safety improvements in the next influenza pandemic. For example, during the COVID-19 pandemic, AI and big data described the disease, improved drug research, identified infected patients and antibodies and tracked the COVID-19 outbreak; however, AI has much greater capacity (Pham et al., 2020). If healthcare organisations prepared AI technology to reduce the impact of the next pandemic, that could improve healthcare safety. According to
Ganasegeran and Abdulrahman (2020), AI is created from three types of different AI concepts, as shown in Figure 2.

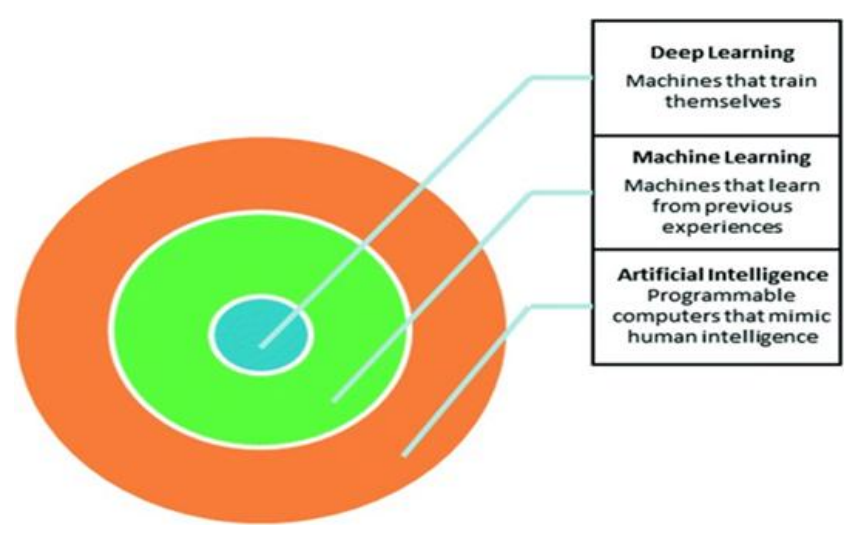

Figure 2: Diagram of main subsets of AI (Ganasegeran et al., 2020)

The second subfield is machine learning (ML), which aims to learn from the past experiences of complex problems. The ML process explores ways to solve issues. It is divided into three categories: supervised learning, unsupervised learning, and reinforcement learning (see Appendix 1) (Ganasegeran and Abdulrahman, 2020). Complex ML systems could find solutions for the next influenza pandemic and help to improve safety in healthcare by tracking the spread of the infection. When healthcare workers apply an early pandemic detection from a computing tool, early reporting from patient zero and the first group of patients and sharing these big data could create a massive development (Allam and Jones, 2020). Moreover, the WHO has highlighted how important it is to share pandemic data (WHO, 2017). There are two successful companies, BlueDot and Metabiota, using AI for big data analysis for airline ticketing and tracking flight data to predict risk areas (Allam and Jones, 2020). For example, the Metabiota realise prediction infection disease by AI, ML, and other technologies using algorithms to collect huge social media data from unofficial and official sources, then this data use to predict human behaviour. While the BlueDot used to predict real-time climate, human disease alert and animal disease by AI and ML technologies. However, implementing both companies of gathering and predicting data in healthcare system from social media and from real-time action, would reduce the impact of the next pandemic. That seems to mean that we have the ability to improve safety before the pandemic becomes a pandemic; ignoring the potential of technology to predict the risk is neglectful.

Spreading and transmitting the virus from an epidemic to pandemic is a complex system and it needs a complex process to solve it, like AI system of dealing with big data. AI and ML provide a better understanding of infectious pandemic prediction, and also collecting and sharing big data will protect the users' information (Agrebi, and Larbi, 2020). The easiest way to monitor public symptoms and infection and identify quick initial diagnosis is using AI mobile apps, such as the recently launched Apple health check app, Siri give me guidance and Alexa daily check (Kapoor et al., 2020). Improving the safety culture protects patients and allows the risk to be assessed. Using these apps could make a significant improvement to the safety culture.

In order to adapt complex systems in the modern world, mobile apps should be used for checking health regularly and providing symptom information to increase the ability to control the next pandemic from an early stage. A similar interpretation of the origins of $\mathrm{AI}$ is Public Health Intelligence (PHI), which can be 
found in French and Mykhalovskiy (2013). To control the disease, the WHO encourages healthcare workers to collect information relating to events, diseases, or any health crisis, even in a small population, to assess the potential risk and reduce spread (WHO, 2005). It is clear that engaging the public is helpful for controlling upcoming infections through the most used devices.

However, the key idea of complexity theory stems from two principles: leverage, making significant developments with small changes; and temporal effects, short-term solutions that cause most of the problems today. This concept is called dynamic complexity (Langley et al., 2009). The health check app is a small change but when implemented right it will lead to a significant improvement, encouraging different aged people to use the app regularly is a long-term solution. Floridi (2020) argued that understanding the nature of the problem in the physical environment means understanding AI's future. Moreover, AI in the form of the mobile app helps workers to meet healthcare infection goals by analysing the existing data and data patterns to predict future outbreaks and control the pandemic. Using COVID-19 as an example, Figure 3 shows the proposed framework of the mobile app (Vanathi and SriPradha, 2020). The thought identifier, alerter and predictor are the basic elements to achieve complex system improvement.

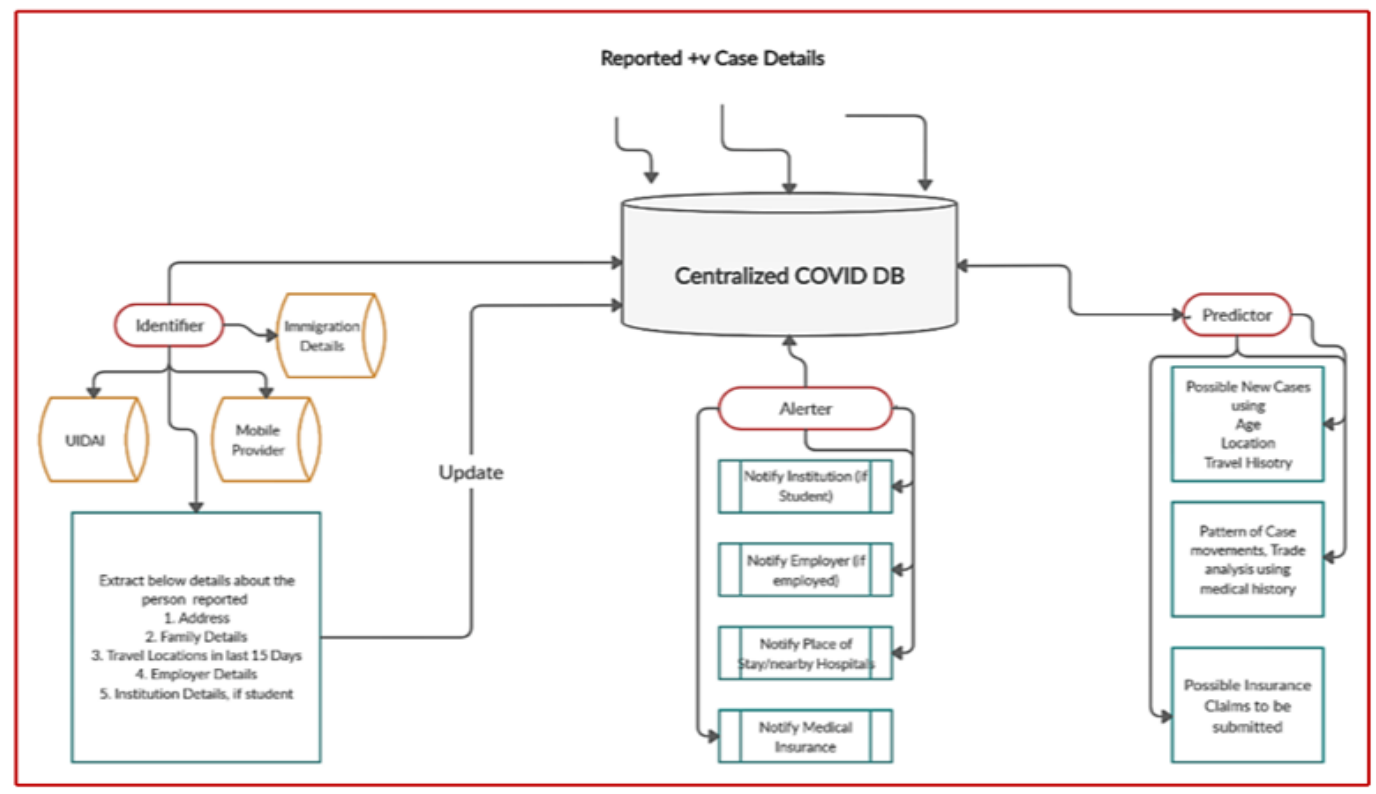

Figure 3: Framework of proposed mobile app to break the Chain (Vanathi and Sri Pradha, 2020).

'Complexity theory indicates that large populations of units can self-organize into aggregations that generate pattern, store information, and engage in collective decision-making' (Parrish and Edelstein-Keshet 1999:99).

\section{Safety Improvement of Central Alerting System}

How to achieve safety improvement is a challenge; other challenges of $\mathrm{AI}$ are responding and analysing the right information at the right time when the system is alert to new infections. French and Mykhalovskiy (2013) indicate that creating and implementing a network alert can improve healthcare systems and the greatest use of this information is protecting the registered diverse informants. From the concept of Safety-II come the proactive safety management principles, which comprise a continuous effort to anticipate events and developments (Hollnagel et al., 2015). If the mobile app is connected to the healthcare alert system, it could predict and issue alerts for expected diseases or infections to prepare action or research into a drug. One of the aspects of AI is that the central alert system is based on protector data, which is automatically monitoring and providing a measurable method (Cho et al., 2020). The process mapping of an initial measure alert is shown in Appendix 2 (Cho et al., 2020).

The alert managing system escalates, notifies, and suppresses different types of data based on predictive and adaptive ML. It also includes user behaviour, dynamic system behaviour, event classification and modelling (Rizk et al., 2020). Recognising the best management for each patient depends on their characteristics, with the most effective policies using available observational data from individuals and evaluating existing treatments (Van der Schaar and Alaa, 2020). For example, patients who have experiences from different management plans from started the system, answering counterfactual questions these can help identify the best measurement plan (Van der Schaar and Alaa, 2020). It is not easy to identify the best measurement plan for predicting a pandemic before it spreads or to analyse all the information, but it is worth the effort to find treatments that can help the healthcare environment through this complex system.

The individual is the important component of having an accurate and effective alert system in which to measure safety, collecting data from mobile apps or other AI technologies is based on individual credibility. As each patient is different, answering questions about their experiences and diagnosis requires individual trueness (Van der Schaar and Alaa, 2020). The individual could be one patient, workers or groups of patients who have a common sense, or even a culture or country. Both public health policy and individuals who use the health check app should be informed of alerts (Parker et al., 2020). Safety can be improved by applying the central alert system to announce the various predictions, which will protect the public and the hospital environment before the event happens. To improve safety complex systems, healthcare providers must ask: What did we do right in the last pandemic? How do we develop it? The right answers should consider the risk from the development viewpoint or positive perspectives. 


\section{Risk Aspects}

The WHO indicated that communication risk is one of the most significant challenges in national plans for facing pandemic influenzas (WHO, 2017). The effect of risk communication strategy is to increase public awareness, which plays a key role in building public confidence and trust in facing the pandemic (Wignjadiputro et al., 2020). The role of regulators in health is to appreciate the risk of communication and provide clear information about facing the pandemic from a trusted source to decrease panic and increase the public's response to AI (Husnayain et al., 2020). It is clear that engaging the public to use AI correctly and also gaining their trust and credibility are critical points. However, the risk of privacy and trust breaches from AI when the public enter their information has three principles: to develop the users' trust that their data will be kept private, avoid surveillance; and only allow one-way private data application (Berke et al., 2020). The risk of communication is related with privacy and distrust somehow, when implementing $\mathrm{AI}$ and ML technology to control the complex issue of the next pandemic. To organise an organisation that is grateful for facing the risk aspects to develop safety, we have to evaluate the risk expected in order to limit the complex problem of the spread of the next influenza pandemic by AI complex theory.

Turchin and Denkenberger (2020) claimed that the risk related to AI of a complex system is linked with a hypothetical scenario called 'paperclip maximiser', which is extinction risks or AI-driven unemployment. They indicated the risk of human-level intelligence and non-self-improvement. Panch et al. (2019) argued that $\mathrm{AI}$ in a low-income community might affect unemployment in the near term, but it has benefits depending on social factors. One question that needs to be asked, however, is whether there is a unique AI system implemented, but without employees this system will never work. Healthcare professionals and hospitals need employee power and the intelligence of the human mind to improve healthcare quality.

Recently, papers have been published on the ethical and challenging risk of implementing an AI complex system to collect data from the public. On the one hand, it could cause some level of privacy infringement, but on the other hand it will reduce suffering and save a huge number of lives (Parker et al., 2020). Ethical big data can limit crisis, for example the COVID-19 situation right now. The world initially started sharing personal data and it has already been collected during this crisis by using $\mathrm{AI}$ in the form of a mobile app (Findlay et al., 2020). The public need to know what will happen to their data, whether it will be deleted or saved after the crisis, and a clear applicable policy would increase their trust in sharing personal data (Parker et al., 2020). According to Panch et al. (2019), there is a possibility that healthcare workers will neglect public involvement after they have collected the data for the system. So, collecting personal data from the public and the idea of increasing the sense of responsibility that provide the public the right of involving process in a wider stage. In order to improve preparation for the next pandemic, these data could be saved from international organisations for developing healthcare.

The non-linear psychological impact on the public of sharing accurate data and being influenced by information comes from different public behaviours and beliefs, which means that different users enter their data with different instructions (Stanley et al., 2020). As suggested, some psychological questions when collecting complex data could help to recognise individual situations.
The financial complexity risk and budget limitations must be well planned because we cannot avoid pandemic effects in worldwide healthcare. A financial plan should be prepared to protect and limit the spread of diseases by improving healthcare quality and safety, which will decrease the financial impact after the pandemic has spread. The impact on businesses and manufacture will affect the economy of individual countries, as has happened in the COVID-19 crisis (Schoenfeld, 2020).

\section{Conclusion}

This essay explored creating a complex safety culture by implementing AI to achieve a scientific breakthrough in safety improvement. It also explored the case of how complexity theory can help safety improvement or mitigate risk in the next influenza pandemic through AI non-linear technologies, with ML playing a significant part. The Deming system explored the appreciate system of complexity from profound knowledge. Safety-II is the mean concept of the complex system, which is non-linear; we ask the question how can we improve; not how can we fail? Employing $\mathrm{AI}$ and the deeper approach of ML will manage a pandemic crisis before it has spread. The world has started to implement the mobile health check app as a kind of AI to control the situation from an initial outbreak in China to identify, predict and alert. The essay also discussed the central alerting system to improve safety and evaluate the risk from different risks of communication, unemployment, ethical, psychological impact, and financial risk aspects.

The unique technology of AI shares worldwide data to help safety improvement in the case of the next pandemic. AI is not just a mobile health check app; it could be other technology. The world and individual countries were badly prepared for the pandemic and the impact of the COVID-19 crisis (Parker et al., 2020). However, organisation is the key to improvement by addressing AI criteria and how we solve the psychological impact while collecting data. These risk aspects must be taken into consideration to achieve the best organisation with the best improvement in facing a pandemic.

There is a slight gap between work as imagined and work as done or practice. To what extent can healthcare workers provide the information and involve the public, will it meet their expectations or affect their trust? How long can we save big data and be protected?

\section{Ethics approval and consent to participate}

This study included no participants and there was no need for either ethical approval or consent.

\section{List of abbreviations}

Artificial Intelligence (AI)

Complex Adaptive System (CAS)

World Health Organization (WHO)

machine learning (ML)

Public Health Intelligence (PHI)

\section{Data Availability}

Data are available upon reasonable request.

\section{Conflicts of Interest}


Author has no conflict of interest

\section{Funding Statement}

None

\section{Authors' contributions}

The work is done by the author of the manuscript.

\section{Acknowledgments}

Author would like to thank everyone who helped completing this manuscript.

\section{References}

[1] Agrebi, S., \& Larbi, A. (2020). Use of artificial intelligence in infectious diseases. In Artificial intelligence in precision health (pp. 415-438). Academic Press.

[2] Allam, Z., Dey, G., \& Jones, D. S. (2020). Artificial Intelligence (AI) provided early detection of the coronavirus (COVID-19) in China and will influence future urban health policy internationally. AI, 1(2), 156165.

[3] Berke, A., Bakker, M., Vepakomma, P., Raskar, R., Larson, K., \& Pentland, A. (2020). Assessing disease exposure risk with location histories and protecting privacy: A cryptographic approach in response to a global pandemic. arXiv preprint arXiv:2003.14412.

[4] Bullock, S., \& Cliff, D. (2004). Complexity and emergent behaviour in ICT systems.

[5] Cho, H., Czarnecki, A., Fackler, S., Guler, C., Taneja, N., Tobin, D., \& Wong, W. (2020). U.S. Patent Application No. 16/689,915.

[6] Dooley, K. J., Johnson, T. L., \& Bush, D. H. (1995). TQM, chaos and complexity. Human Systems Management, 14(4), 287-302.

[7] Findlay, M., Loke, J. Y., Remolina, N., \& Tham, B. (2020). Ethics, AI, mass data and pandemic challenges: Responsible data use and infrastructure application for surveillance and pre-emptive tracing post-crisis. SMU Centre for AI \& Data Governance Research Paper Forthcoming.

[8] Floridi, L. (2020). What the near future of artificial intelligence could be. In The 2019 Yearbook of the Digital Ethics Lab (pp. 127-142). Springer, Cham.

[9] French, M., \& Mykhalovskiy, E. (2013). Public health intelligence and the detection of potential pandemics. Sociology of Health \& Illness, 35(2), 174-187.

[10] Ganasegeran, K., \& Abdulrahman, S. A. (2020). Artificial intelligence applications in tracking health behaviors during disease epidemics. In Human behaviour analysis using intelligent systems (pp. 141-155). Springer, Cham.

[11] Hmelo-Silver, C. E., \& Pfeffer, M. G. (2004). Comparing expert and novice understanding of a complex system from the perspective of structures, behaviors, and functions. Cognitive Science, 28(1), 127-138.

[12] Hollnagel, E., Wears, R. L., \& Braithwaite, J. (2015). From Safety-I to Safety-II: a white paper. The resilient health care net: published simultaneously by the
University of Southern Denmark, University of Florida, USA, and Macquarie University, Australia.

[13] Husnayain, A., Fuad, A., \& Su, E. C. Y. (2020). Applications of google search trends for risk communication in infectious disease management: A case study of COVID-19 outbreak in Taiwan. International Journal of Infectious Diseases.

[14] Jiang, F., Jiang, Y., Zhi, H., Dong, Y., Li, H., Ma, S., \& Wang, Y. (2017). Artificial intelligence in healthcare: past, present and future. Stroke and Vascular Neurology, 2(4), 230-243.

[15] Kapoor, A., Guha, S., Das, M. K., Goswami, K. C., \& Yadav, R. (2020). Digital healthcare: The only solution for better healthcare during COVID-19 pandemic? Indian Heart Journal.

[16] Ladyman, J., Lambert, J., \& Wiesner, K. (2013). What is a complex system? European Journal for Philosophy of Science, 3(1), 33-67.

[17] Langley, G. J., Moen, R. D., Nolan, K. M., Nolan, T. W., Norman, C. L., \& Provost, L. P. (2009). The improvement guide: a practical approach to enhancing organizational performance. John Wiley \& Sons.

[18] Panch, T., Pearson-Stuttard, J., Greaves, F., \& Atun, R. (2019). Artificial intelligence: opportunities and risks for public health. The Lancet Digital Health, 1(1), e13-e14.

[19] Parker, M. J., Fraser, C., Abeler-Dörner, L., \& Bonsall, D. (2020). Ethics of instantaneous contract tracing using mobile phone apps in the control of the COVID-19 pandemic. Journal of Medical Ethics.

[20] Parker, M. J., Fraser, C., Abeler-Dörner, L., \& Bonsall, D. (2020). Ethics of instantaneous contact tracing using mobile phone apps in the control of the COVID-19 pandemic. Journal of Medical Ethics.

[21] Parrish, J. K., \& Edelstein-Keshet, L. (1999). Complexity, pattern, and evolutionary trade-offs in animal aggregation. Science, 284, 99-101.

[22] Pham, Q. V., Nguyen, D. C., Hwang, W. J., \& Pathirana, P. N. (2020). Artificial Intelligence (AI) and big data for coronavirus (COVID-19) pandemic: A survey on the state-of-the-arts.

[23] Rizk, Y., Isahagian, V., Unuvar, M., \& Khazaeni, Y. (2020). A snooze-less user-aware notification system for proactive conversational agents. arXiv preprint arXiv:2003.02097.

[24] Schoenfeld, J. (2020). The invisible risk: Pandemics and the financial markets. Available at SSRN 3567249.

[25] Stanley, M., Seli, P., Barr, N., \& PETERS, K. (2020). Analytic-thinking predicts hoax beliefs and helping behaviors in response to the COVID-19 Pandemic.

[26] Sturmberg, J. P., \& Martin, C. (Eds.). (2013). Handbook of systems and complexity in health. Springer Science \& Business Media.

[27] Taubenberger, J. K., \& Morens, D. M. (2006). 1918 Influenza: the mother of all pandemics. Revista Biomedica, 17(1), 69-79.

[28] Turchin, A., \& Denkenberger, D. (2020). Classification of global catastrophic risks connected with artificial intelligence. AI \& Society, 35(1), 147-163.

[29] Van der Schaar, M., \& Alaa, A. (2020). How artificial intelligence and machine learning can help healthcare systems respond to COVID-19. 
[30] Vanathi, J., \& SriPradha, G. (2020). Break the chain: A proposed AI powered mobile application framework to handle COVID-19 Pandemic.

[31] Wignjadiputro, I., Widaningrum, C., Setiawaty, V., Wulandari, E. W., Sihombing, S., Prasetyo, W. A., \& Subuh, M. (2020). Whole-of-society approach for influenza pandemic epicentre. Containment exercise in Indonesia. Journal of Infection and Public Health.

[32] World Health Organization (WHO) (2005) International health regulations, 2nd edn. Geneva: WHO.

[33] World Health Organization. (2017). Pandemic influenza risk management - A WHO guidance.

[34] Paley, J., \& Eva, G. (2011). Complexity theory as an approach to explanation in healthcare: A critical discussion. International Journal of Nursing Studies, 48(2), 269-279.

[35] Thompson, D. S., Fazio, X., Kustra, E., Patrick, L., \& Stanley, D. (2016). Scoping review of complexity theory in health services research. BMC health services research, 16(1), 87.
[36] Begun, J. W., Zimmerman, B., \& Dooley, K. (2003). Health care organizations as complex adaptive systems. Advances in health care organization theory, 253, 288.

[37] Greenhalgh, T., Plsek, P., Wilson, T., Fraser, S., \& Holt, T. (2010). Response to 'The appropriation of complexity theory in health care'. Journal of Health Services Research \& Policy, 15(2), 115-117.

[38] Liu, H. (2018). Language as a human-driven complex adaptive system. Physics of life reviews, 26, 149-151.

[39] Yassine, H. M., \& Shah, Z. (2020). How could artificial intelligence aid in the fight against coronavirus? An interview with Dr Hadi M Yassine and Dr Zubair Shah by Felicity Poole, Commissioning Editor. Expert review of anti-infective therapy, 18(6), 493-497.

[40] Liehr, P., \& Smith, M. J. (2017). Middle Range Theory: a perspective on development and use. Advances in Nursing Science, 40(1), 51-63.

[41] Shearer, F. M., Moss, R., McVernon, J., Ross, J. V., \& McCaw, J. M. (2020). Infectious disease pandemic planning and response: Incorporating decision analysis. PLoS Medicine, 17(1). 


\section{Appendices}

\section{Appendix 1}

Table of Common ML methods applicable to track health-behaviors during infectious disease epidemics

\begin{tabular}{|l|l|l|}
\hline Types & Examples of methods used & Functions \\
\hline \multirow{5}{*}{$\begin{array}{l}\text { Supervised } \\
\text { method }\end{array}$} & $\begin{array}{l}\text { Support Vector Machine (SVM)s } \\
\text { Decision Tree, } \\
\text { Random Forest, } \\
\text { Naive Bayes (NB)s } \\
\text { Artificial Neural Network (ANN)s } \\
\text { Bootstrap Aggregatings } \\
\text { AdaBoost }\end{array}$ & $\begin{array}{l}\text { The primary function of these methods is to create prediction outcomes to warn } \\
\text { authorities and public in advance on potential infectious disease epidemics, thus } \\
\text { suggesting immediate prevention and control strategies }\end{array}$ \\
\hline \multirow{3}{*}{$\begin{array}{l}\text { Unsupervised } \\
\text { method }\end{array}$} & \begin{tabular}{l} 
Principal Component Analysis (PCA) \\
\cline { 2 - 3 }
\end{tabular} & $\begin{array}{l}\text { This method aims to reduce data dimensions to enable researchers easily uncover } \\
\text { Key factors causing infectious disease dynamics }\end{array}$ \\
\cline { 2 - 3 } & Latent Dirichlet Allocation (LDA) & This method clusters patients for abnormality detections \\
\cline { 2 - 3 } & Deep Learning Architectures & $\begin{array}{l}\text { This method enables data extraction from medical contextual records } \\
\text { applications of bioinformatics in infectious disease analytics }\end{array}$ \\
\cline { 2 - 3 } & Structural Equation Model Trees & $\begin{array}{l}\text { This method allows estimation of complex cause-effect relationship models with } \\
\text { latent variables }\end{array}$ \\
\hline
\end{tabular}

(Ganasegeran and Abdulrahman, 2020).

Appendix 2:
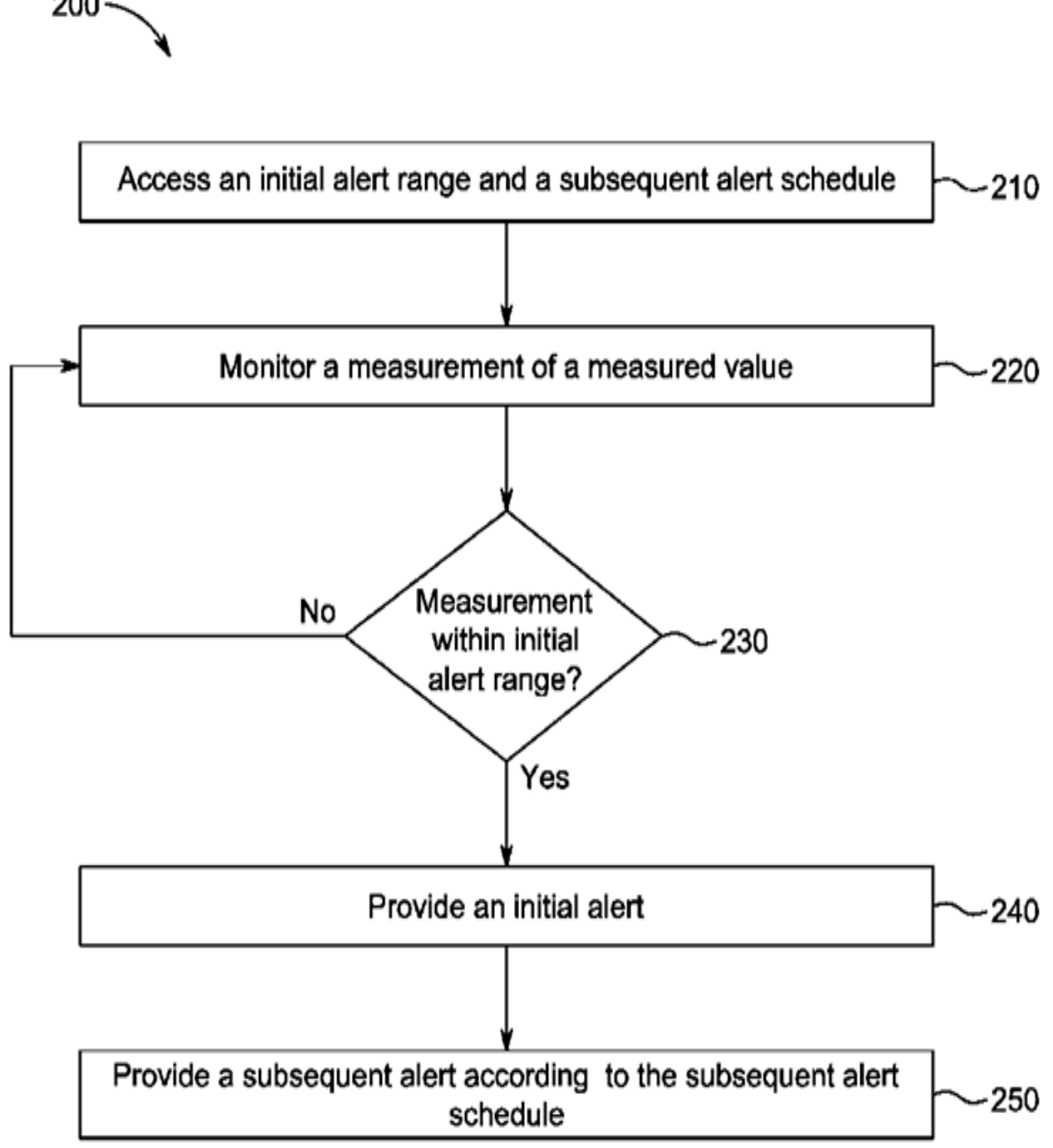

(Cho et al., 2020). 